1,13

\title{
Динамический потенциал взаимодействия атомов азота с поверхностью кристалла алюминия
}

\author{
(C) В.С. Малышевский, Г.В. Фомин, Е.В. Дергачева, Т.И. Жилина
}

Южный федеральный университет,

Ростов-на-Дону, Россия

E-mail: vsmalyshevsky@sfedu.ru

(Поступила в Редакцию 27 декабря 2017 г.)

\begin{abstract}
Методом компьютерного моделирования исследованы особенности угловых распределений ускоренных нейтральных атомов азота при скользящих углах падения на поверхность кристалла $\mathrm{Al}(001)$. Парный потенциал взаимодействия $\mathrm{N}-\mathrm{Al}$ аппроксимировался трехпараметрическим потенциалом Морзе, с зависящими от энергии коэффициентами. Моделирование угловых распределений рассеянных атомов осуществлялось с учетом взаимодействия атомов с несколькими слоями атомов в кристаллической решетке и смещением атомов в процессе тепловых колебаний. Определены параметры парного потенциала ускоренных нейтральных атомов азота в диапазоне энергий от 10 до $70 \mathrm{keV}$ по наилучшему согласию расчетной зависимости радужного угла рассеяния от энергии падающих на поверхность кристалла частиц с имеющимися экспериментальными данными.
\end{abstract}

DOI: $10.21883 /$ FTT.2018.06.46001.361

\section{1. Введение}

Явление радужного рассеяния при скользящих падениях на поверхность кристалла возникает при направлении движения частиц под малым углом вдоль атомных цепочек, когда зависимость азимутального угла рассеяния $\Theta(\rho)$ (т.е. угла в плоскости, расположенной перпендикулярно атомным цепочкам) от прицельного параметра $\rho$ относительно выбранной атомной цепочки имеет экстремумы. Значение этого угла рассеяния $\Theta_{R}$ в максимумах углового распределения рассеянных частиц называют радужным углом (см. рис. 1). Экспериментальному и теоретическому исследованию этого эффекта посвящено несколько недавних работ, например [1-5]. Как отмечается в цитированных работах, по угловым распределениям рассеянных частиц можно судить о структуре поверхности и характере потенциала взаимодействия между рассеиваемыми атомами и поверхностью кристаллов. Следует отметить, что эффекты радужного рассеяния наблюдаются и в экспериментах на прохождение заряженных частиц через кристаллы [6]. Хотя геометрия рассеяния в этом случае иная, но, как и при скользящих падениях на поверхность кристалла, радужный эффект и в этом случае обусловлен немонотонной зависимостью угла рассеяния от прицельного параметра.

Интересным явлением, наблюдаемым в экспериментax $[1,2]$, является зависимость от энергии углов радужного рассеяния нейтральных атомов поверхностью металлических и диэлектрических кристаллов. В частности, в [2] установлено, что для атомов азота и кислорода зависимость угла радужного рассеяния от начальной поперечной энергии (или иначе от поперечной компоненты скорости, т.е. нормальной к поверхности кристалла) может иметь как монотонный характер при больших энергиях частиц, так и немонотонный характер при малых энергиях частиц. Причем в случае рассеяния от поверхности металлического кристалла Al увеличение полной кинетической энергии частиц сопровождается уменьшением радужного угла при фиксированном значении поперечной энергии. В случае же рассеяния атомов поверхностью диэлектрического кристалла $\mathrm{LiF}$ при таких же условиях значение радужного угла не зависит от полной кинетической энергии частиц.

Очевидно, что наблюдаемые динамические явления в рассеянии могут быть связаны с диссипативными процессами, а именно, с зависимостью взаимодействия погруженного в электронный газ атома с поверхностью металлического кристалла от его кинетической энергии. А именно, описанные закономерности можно объяснить влиянием электронного торможения медленных атомных частиц вблизи поверхности металлического кристалла. Действительно, в цитированных эксперименталь-

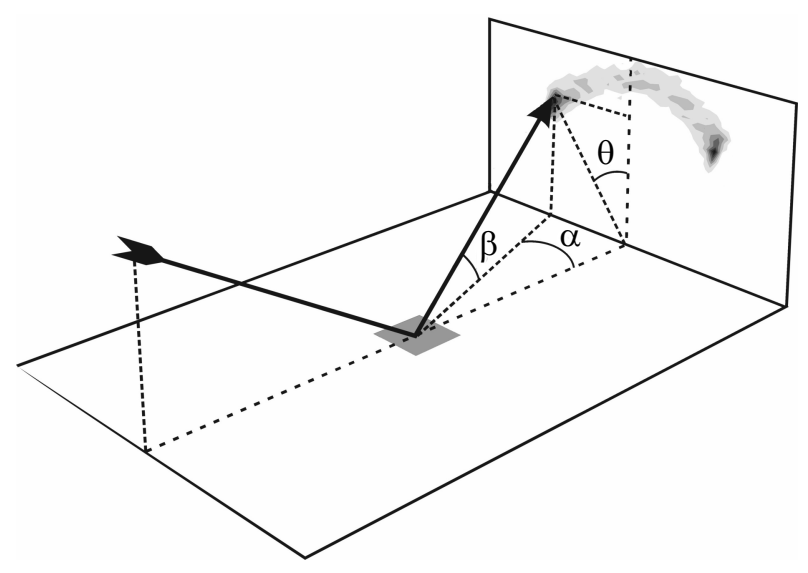

Рис. 1. Геометрия рассеяния атомов поверхностью кристалла при скользящих падениях. 
ных работах скорость рассеваемых атомных частиц не превышает фермиевскую скорость (например, при энергии атомов азота $10 \mathrm{keV}$ их скорость равна $3.7 \cdot 10^{7} \mathrm{~cm} / \mathrm{s}$, в то время как фермиевская скорость электронов в $\mathrm{Al}$ на порядок больше и равна $2.03 \cdot 10^{8} \mathrm{~cm} / \mathrm{s}$ ). В этом случае на характер взаимодействия медленной атомной частицы с электронной подсистемой существенную роль оказывает статистика Ферми электронного газа и величина скорости частицы. При скоростях, намного меньших фермиевской, передача импульса электронам настолько мала, что только для электронов из небольшого слоя вблизи поверхности Ферми есть возможность перейти в свободные состояния. Увеличение скорости и, соответственно, переданного импульса приводит к увеличению этого слоя и, следовательно, к увеличению числа электронов, вовлеченных в процесс передачи импульса. Вследствие этого значение тормозного сечения возрастает вместе со скоростью частицы $[7,8]$. Как показано в $[3,4]$, именно это обстоятельство является причиной уменьшения радужного угла с увеличением скорости частицы. С другой стороны, описание атомных столкновений при малых скоростях сталкивающихся частиц можно проводить, используя зависящие от скорости парные потенциалы [9]. Зависимость потенциалов от скорости появляется при учете в уравнениях ТомасаФерми-Дирака передачи кинетической энергии электронам сталкивающихся атомов. В этом случае статистика Ферми также определяет доступное число состояний электронной подсистемы, вовлеченной в передачу энергии. Оба подхода в определенной степени эквивалентны, поскольку и в том и другом случае речь идет о передаче энергии электронной подсистеме, а область разрешенных начальных состояний определяется скоростью относительного движения сталкивающихся атомных частиц.

В данной работе продемонстрирована возможность восстановления парного динамического потенциала по зависимости радужного угла рассеяния атомов азота при скользящих падениях на поверхность кристалла алюминия от полной кинетической энергии атомных частиц. Результаты компьютерного моделирования, с использованием найденного динамического потенциала для взаимодействия $\mathrm{N}-\mathrm{Al}$, дают хорошее согласие с экспериментальными данными.

\section{2. Описание модели взаимодействия}

Процесс рассеяния пучка моделировался численным интегрированием методом Дормана-Принса [10] классических нерелятивистских уравнений движения каждой отдельной частицы в поле поверхности кристалла алюминия с учетом двухчастичных потенциалов взаимодействия. Учитывалось поле, созданное ближайшим окружением с выбранным числом атомов и слоев кристаллической поверхности. Положение атомов кристалла определялось известной структурой кристаллической решетки алюминия и дисперсией нормального распреде- ления тепловых смещений. При этом каждая отдельная частица пучка на протяжении длины пробега (порядка нескольких постоянных решетки) испытывала влияние поля неподвижных атомов, находящихся в случайно смещенных положениях. Для фиксации выходных параметров рассеянных частиц использовались полярные координаты в соответствии с рис. 1, на котором $\alpha-$ так называемый выходной азимутальный угол, а $\beta-$ выходной полярный угол. Разработанное приложение позволяет прослеживать характер траекторий рассеиваемых частиц, а также зависимость выходных параметров от прицельного параметра для отдельных траекторий. Для набора достаточной статистики накопление данных осуществлялось для 20000 траекторий.

Потенциал взаимодействия налетающего атома с ориентированной кристаллической поверхностью $U(\mathbf{r})$ определим как суперпозицию потенциалов взаимодействия с отдельными атомами (ионами) поверхности в первом и последующих слоях:

$$
U(\mathbf{r})=\sum_{j} V_{a}\left(\left|\mathbf{r}-\mathbf{r}_{j}\right|\right) .
$$

Взаимодействия нейтральных атомов $\mathrm{N}$ с поверхностью кристалла $\mathrm{Al}(001)$ при скользящих падениях будем описывать потенциалом Морзе, который является известной аналитической трехпараметрической аппроксимацией [11]

$$
V_{a}(r)=\varepsilon\{\exp [-2 \alpha(r-\sigma)]-2 \exp [-\alpha(r-\sigma)]\} .
$$

Как показано в [5], аппроксимация Морзе (2) хорошо описывает рассчитанный на основе теории функционала электронной плотности статический потенциал в широком диапазоне прицельных параметров.

На угловое распределение радужного рассеяния оказывают влияние структурные особенности, связанные с релаксацией и реконструкцией поверхности. Нормальная релаксация наблюдается в металлах и в большинстве случаев имеет место уменьшение первого межслоевого расстояния. Эти и другие особенности учтены при построении модели взаимодействия и рассеяния атомных частиц поверхностью кристалла. Как следует из проведенных ранее расчетов методом функционала электронной плотности [5], расстояние между первым и вторым слоем плоскости (001) монокристалла Al в результате нормальной релаксации составляет $1.743 \AA$, тогда как в объеме расстояние между ближайшими слоями равно $2.025 \AA$. Это обстоятельство приводит к изменению потенциального рельефа, на котором рассеиваются падающие на поверхность частицы, и к изменению характера их траекторий и угловых распределений.

В настоящей работе путем моделирования траекторий частиц рассмотрены особенности отражения нейтральных атомов азота от поверхности кристалла $\mathrm{Al}$ (001) при скользящих падениях. Сравнение результатов расчетов с экспериментальными данными позволило уточнить параметры аналитической аппроксимации парного потенциала взаимодействия атомных частиц. 
Значения параметров парного потенциала Морзе $\mathrm{N}-\mathrm{Al}$ для различных энергий атомов азота

\begin{tabular}{c|l|l|l|l}
\hline$E, \mathrm{keV}$ & 10 & 25 & 40 & 70 \\
\hline$\varepsilon, \mathrm{eV}$ & 0.41 & 0.35 & 0.30 & 0.25 \\
$\sigma, \AA$ & 2.76 & 2.95 & 3.10 & 3.30 \\
$\alpha, 1 / \AA$ & 1.0 & 1.0 & 1.0 & 1.0
\end{tabular}

\section{3. Результаты компьютерного моделирования}

Зависимость угла радужного рассеяния от начальной поперечной энергии для атомов азота оказывается принципиально различной в зависимости от полной кинетической энергии атомов. Угол радужного рассеяния при увеличении энергии уменьшается. Наилучшее согласие с экспериментальными данными [2] достигается при динамических параметрах потенциала Морзе, приведенных в таблице и на рис. 2.

Некоторые результаты моделирования с использованием потенциала (2) показаны на рис. 3 и 4. Угловые распределения при начальной поперечной энергии $12 \mathrm{eV}$ (рис. 3) полностью согласуются с экспериментальными данными [2]. Зависимость радужного угла от поперечной энергии при различных полных энергиях иллюстрируется на рис. 5. В широком интервале поперечных энергий наблюдается неплохое согласие с экспериментальными данными [2]. Следует отметить, что при энергии атомов азота $70 \mathrm{keV}$ не удается добиться согласия с экспериментальными данными во всем интервале поперечных энергий с использованием аппроксимации Морзе. Результаты компьютерного моделирования в потенциале Морзе дают несколько завышенный результат для радужного угла при малых поперечных энергиях (или, что то же самое, при больших прицельных параметрах). Для качественно-

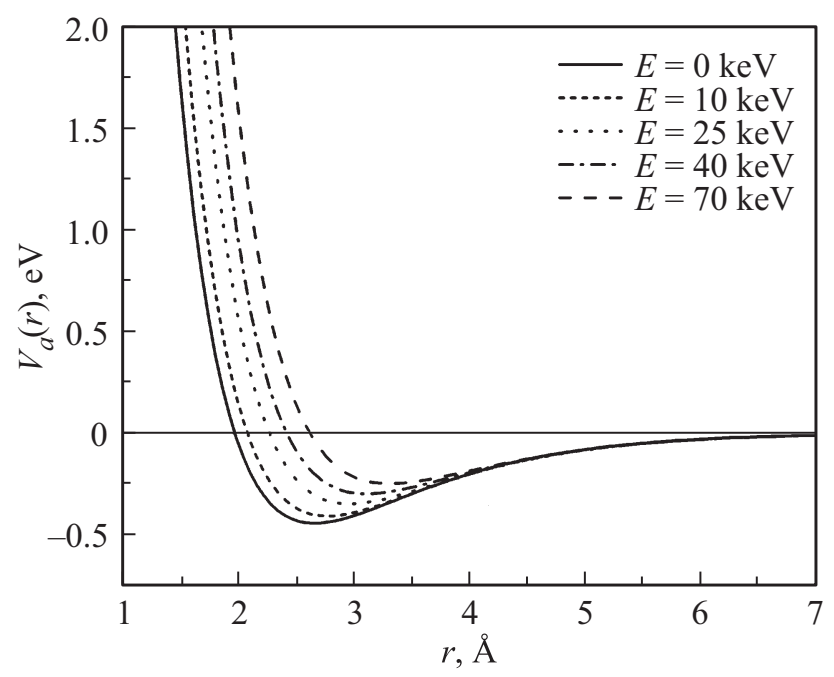

Pис. 2. Динамический парный потенциал $\mathrm{N}-\mathrm{Al}$ в аппроксимации Морзе при различных значениях энергии.

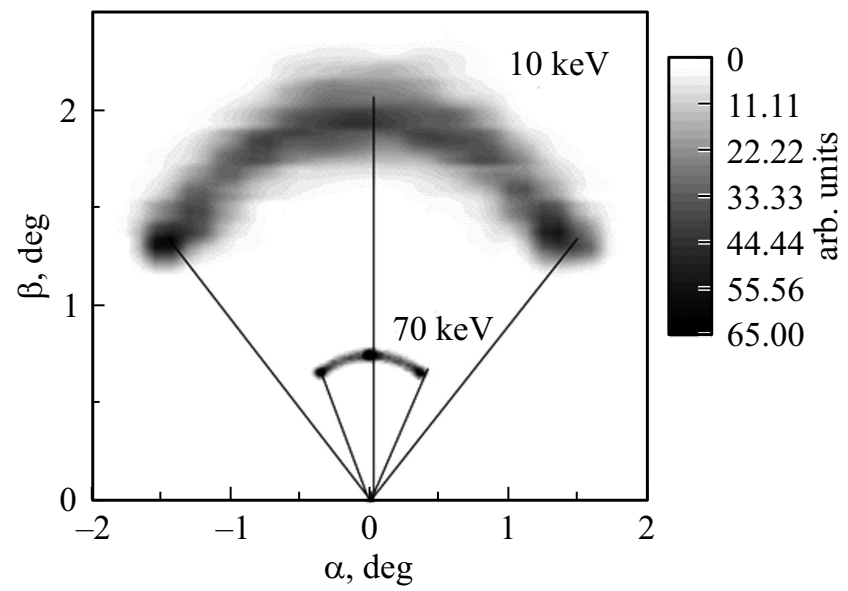

Рис. 3. Результаты компьютерного моделирования углового распределения рассеянных нейтральных атомов азота с энергиями 10 и $70 \mathrm{keV}$ в полярных координатах при скользящем падении налетающих частиц на поверхность (001) кристалла $\mathrm{Al}$ вдоль осевого направления $\langle 100\rangle$. Начальная поперечная энергия $12 \mathrm{eV}$.

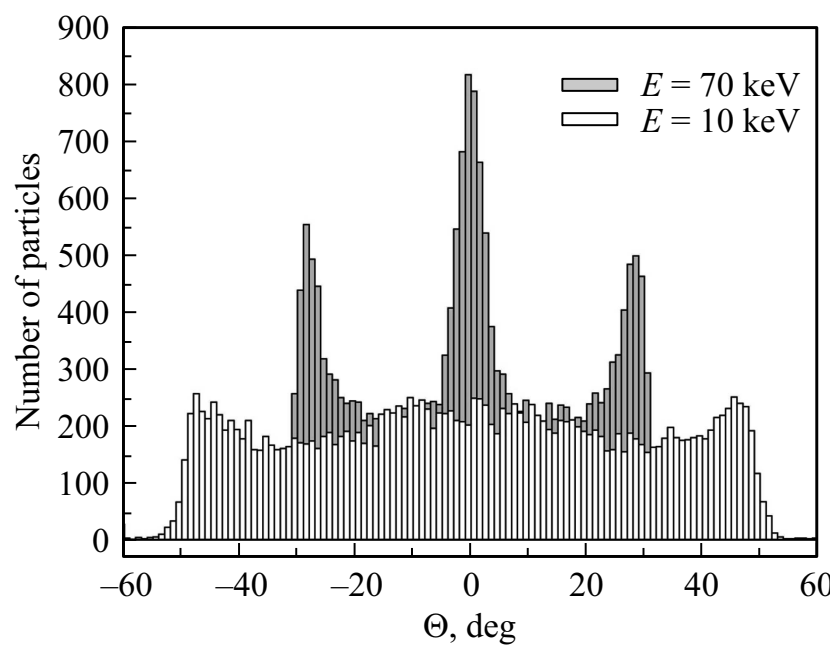

Рис. 4. Рассчитанные зависимости от азимутального угла распределений рассеянных атомов азота поверхностью (001) кристалла Al при скользящем падении налетающих частиц с энергией 10 и $70 \mathrm{keV}$ вдоль осевого направления $\langle 100\rangle$ с поперечной энергией $12 \mathrm{eV}$.

го понимания этого результата заметим, что при энергии атомов азота $70 \mathrm{keV}$ их скорость равна $0.97 \cdot 10^{8} \mathrm{~cm} / \mathrm{s}$, что уже сравнимо с приведенной выше фермиевской скоростью. Это означает, что в процесс передачи импульса электронной подсистеме вовлечены все заполненные состояния и тормозное сечение перестает возрастать с увеличением скорости атомной частицы. Зависимость потерь энергии от скорости определяется сечением рассеяния на короткодействующем потенциале, которое уменьшается с увеличением скорости частицы. С точки зрения потенциального рассеяния это свидетельствует о том, что используемая аппроксимация потенциала 


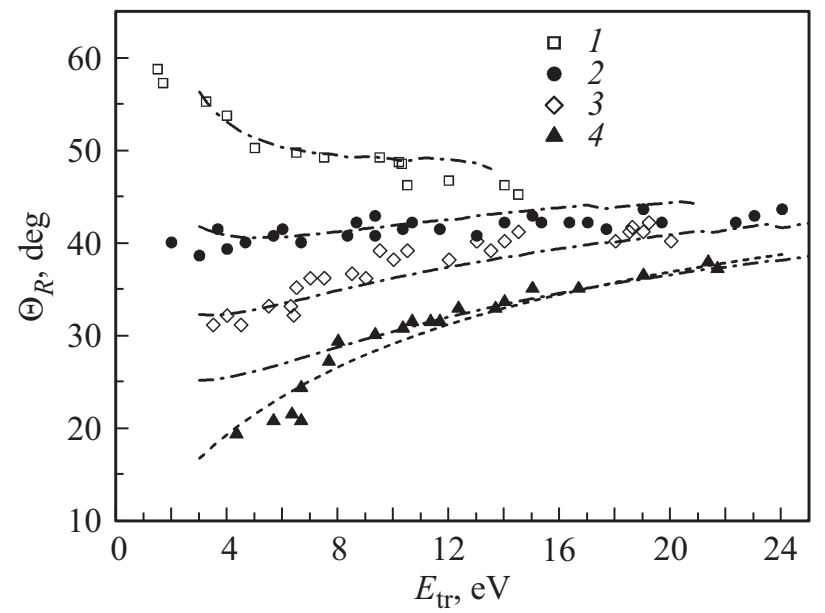

Рис. 5. Экспериментальные данные [2] зависимости радужного угла рассеяния нейтральных атомов азота от значения поперечной энергии при падении налетающих частиц с различными энергиями: $1-10 \mathrm{keV}, 2-25 \mathrm{keV}, 3-40 \mathrm{keV}, 4-70 \mathrm{keV}$ на поверхность (001) кристалла Al вдоль осевого направления $\langle 100\rangle$. Соответствующие результаты моделирования при таких же значениях энергии показаны штрихпунктирными линиями. Пунктирная линия - расчет радужного угла при энергии $70 \mathrm{keV}$ в парном потенциале О’Коннора-Бирсака.

\section{Список литературы}

[1] A. Schuller, H. Winter. Nucl. Inst. Meth. Phys. Res. B 256, 122 (2007).

[2] A. Schuller, H. Winter. Nucl. Inst. Meth. Phys. Res. B 267, 628 (2009).

[3] В.С. Малышевский, А.В. Казаков. Письма в ЖТФ 37, 17, 98 (2011).

[4] V.S. Malyshevsky. Nucl. Instr. Meth. Phys. Res. B 309, 151 (2013).

[5] Л.А. Авакян, Т.И. Жилина, В.С. Малышевский, Г.В. Фомин. Поверхность. Рентгеновские, синхротронные и нейтронные исследования 1, 80 (2016).

[6] H.F. Krause, S. Datz, P.F. Ditter, J. Gomes del Campo, P.D. Miller, C.D. Moak, N. Neskovic, P.L. Permiller. Phys. Rev. B 33, 6036 (1986).

[7] E. Fermi, E. Teller. Phys. Rev. 72, 399 (1947).

[8] И.А. Ахиезер, Л.Н. Давыдов. УФН 129, 239 (1979).

[9] Г.В. Дедков. УФН 165, 919 (1995).

[10] J.R. Dormand, P.J. Prince. J. Comp. Appl. Math. 6, 1, 19 (1980).

[11] L.A. Girifalco, V.G. Weizer. Phys. Rev. 114, 687 (1959).

[12] D.J. O’Connor, J.P. Biersack. Nucl. Instr. Meth. Phys. Res. B 15, 14 (1986).

Морзе несколько недооценивает роль экранирования атомных потенциалов при малых поперечных энергиях и при скоростях, сравнимых или больше фермиевской скорости. Действительно, меньший угол рассеяния свидетельствует о меньшей силе, действующей на частицу, или иначе, большей степени экранирования атомных потенциалов. В этом случае потенциал взаимодействия, описывающий процесс рассеяния, будет являться потенциалом отталкивания, который определяется взаимодействием ядер и электронов внутренних оболочек атомов. В частности, проведенное моделирование процесса рассеяния с использованием потенциала Томаса-Ферми в аппроксимации О’Коннора-Бирсака [12] дает наилучшее согласие с измеренными данными для энергии атомов $70 \mathrm{keV}$ (см. рис. 5) во всем интервале поперечных энергий.

\section{4. Заключение}

Показана возможность описания особенностей радужного рассеяния атомных частиц поверхностью металлических кристаллов при скользящих падениях с использованием динамического потенциала в аппроксимации Морзе. С помощью компьютерного моделирования определены параметры парного потенциала взаимодействия ускоренных нейтральных атомов азота в диапазоне энергий от 10 до $70 \mathrm{keV}$ по наилучшему согласию расчетной зависимости радужного угла рассеяния от энергии падающих на поверхность кристалла алюминия частиц с имеющимися экспериментальными данными. 